\title{
Sistema informático para la gestión de ensayos fisicoquímicos en la Central Termoeléctrica Ernesto Guevara
}

DOI: https://doi.org/10.33262/ap.v2i2.27

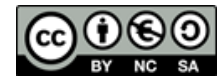

Computer system for the management of physicochemical tests at the Ernesto Guevara Thermoelectric Power Plant

Naddiel Jesús Sánchez Rojas., ${ }^{1}$ Amaury Machín Armenteros. ${ }^{2}$ \& Yusney Marrero García. ${ }^{3}$

\begin{abstract}
.
The management of information associated with the physicochemical tests carried out in the Department of Chemistry in the Thermoelectric Plant "Ernesto Guevara" is affected since it is carried out through text documents and spreadsheets. This leads to bad exploitation of the data and implies the generation of large volumes of information, which are difficult to save and process, and hence, hinder the control of electricity generation. Therefore, the process of supervision and control of the electricity generation is hindered. For these reasons, it was decided to develop a computer system that improves the information management. As a result of the present work, a system was obtained that allows the management and control of the information, which allows a better organization, quick answers to the queries, and precise data that can be used as support in the decision-making process in the Department of Chemistry.
\end{abstract}

\footnotetext{
${ }^{1}$ Investigador

${ }^{2}$ Investigador

${ }^{3}$ Investigador
} 
Keywords: computer management, physicochemical tests, data exploitation.

\section{Resumen.}

La gestión de la información asociada a los ensayos fisicoquímicos que se llevan a cabo en el Departamento de Química de la Central Termoeléctrica Ernesto Guevara se ve afectada debido a que dicha gestión se realiza a través de documentos de textos, y hojas de cálculo. Esto impide una buena explotación de los datos e implica que se generen grandes volúmenes de información, difíciles de guardar y procesar, lo cual dificulta el control del proceso de generación eléctrica. Por estas razones se decide desarrollar un sistema informático que mejore la gestión de información. Como resultado del presente trabajo se obtuvo un sistema que permite la gestión y control de la información, lo cual garantiza una mayor organización, respuestas rápidas ante las consultas, y datos precisos que podrán ser utilizados como apoyo en el proceso de toma de decisiones en el Departamento de Química.

Palabras claves: gestión informática, ensayos fisicoquímicos, explotación de datos.

\section{Introducción.}

A lo largo de la historia la energía ha jugado un papel fundamental en el desarrollo del hombre. Primero el fuego, usado para la cocción y la iluminación, hasta que apareció la energía eléctrica como fuente principal. La generación de energía de forma segura y sistemática tomó lugar en la vida del hombre en el siglo XIX. En la actualidad existen muchas formas de generar energía eléctrica. Desde las fuentes limpias, como el aire y el sol hasta los combustibles fósiles y las fuentes radioactivas. En nuestro país la forma más difundida de generar energía es a través del uso de combustibles fósiles, específicamente petróleo, en las termoeléctricas.

Dentro de las centrales termoeléctricas (CTE) los laboratorios químicos juegan un papel fundamental en la generación de energía de forma eficaz y segura. Su trabajo está centrado en la realización de ensayos que permiten obtener resultados indispensables para la toma de decisiones. Dentro de los diferentes ensayos que se realizan en los laboratorios están aquellos dirigidos a evaluar las características fisicoquímicas de los combustibles y lubricantes, del agua en el ciclo agua-vapor, así como en la producción de agua desmineralizada, la cual es imprescindible para el proceso de generación eléctrica.

La generación de electricidad es el proceso de producir energía eléctrica a partir de otras fuentes de energía primaria. Esta es considerada la espina dorsal del desarrollo de cada país. Los principios fundamentales de la generación de electricidad fueron descubiertos durante las décadas de 1820 y principios de 1830 por el científico británico Michael Faraday (Mansoor-ul; 2019). 
Las plantas de energía alimentada con combustibles fósiles (FFPP, por sus siglas en inglés) son un grupo de dispositivos de generación de energía que convierten la energía química almacenada en el combustible fósil, como el carbón, el gas, o el petróleo en energía térmica, energía mecánica y finalmente energía eléctrica. En los últimos cien años, las FFPP son las fuentes más utilizadas en la industria de la energía, y desempeñan un papel fundamental en la producción y la vida social. Para la mayor parte del mundo la generación de electricidad a través de las FFPP no cambiará en el futuro próximo. De acuerdo a las estadísticas mundiales de energía de 2013 publicadas por la Agencia Internacional de Energía (AIE), en 2011, el $68 \%$ de toda la energía generada provenía de fuentes de este tipo.

La esencia del proceso de producción de energía a través de las FFPP es la conversión de energía. En la gran mayoría de las FFPP, el agua / vapor se utiliza comúnmente como el fluido de trabajo, que se vaporiza alternativamente, y es condensado en un circuito cerrado siguiendo un ciclo termodinámico. Dentro de este ciclo, la energía química del combustible fósil se transforma en energía de vapor térmico por la caldera, luego en energía mecánica rotacional por la turbina, y finalmente en energía eléctrica por el generador (Jiong, 2015). El 20 de febrero de 1966 entraron en servicio en Cuba dos generadores, de la Unión Soviética y de la República de Checoslovaquia, los cuales se instalaron en las dos primeras unidades de las Centrales Termoeléctricas Antonio Maceo (Renté) y Máximo Gómez (Mariel). El comandante Ernesto Che Guevara dio un importante impulso a todos estos planes, pues durante su etapa en el Ministerio de Industrias se firmaron estos contratos (ECURED; 2018).

La empresa mixta ENERGAS, cuenta con 7 instalaciones para la generación de electricidad mediante turbinas de gas y ciclos combinados, en las provincias de Matanzas y Mayabeque.

En la actualidad en nuestro país La Unión Eléctrica está integrada por ocho centrales termoeléctricas, radicadas en las provincias de Mayabeque, La Habana, Matanzas, Cienfuegos, Camagüey, Holguín y Santiago de Cuba y una Organización Básica Eléctrica en cada una de las provincias (4).

Las propuestas detectadas son utilizadas para propósitos generales en departamentos de química. Entre los encontrados se destacan:

- VelocityEHS, Chemical Management Solution (Solución de Gestión Química); Es un software de gestión múltiple, que tiene como propósito general el manejo de inventarios químicos, gestión segura de hojas de datos, reportes regulares y etiquetado personalizado. Requiere conexión a internet todo el tiempo para el manejo de datos y necesidad de pago (www.ehs.com).

- Intelex, Chemical Inventory Management Software (Software de gestión de inventario químico); Permite recopilar, almacenar e informar sobre todos los datos que necesitan para una gestión eficiente de sustancias químicas y materiales peligrosos. Las principales funcionalidades son: Monitoreo de ubicación de 
productos químicos riesgosos a través de toda la organización, historial sobre tendencias y usos de productos químicos, y generación fácil de inventarios químicos (www.intelex.com).

A pesar de que los sistemas antes descritos son usados en departamentos de química no pueden ser empleados en el Área Química de la Central Termoeléctrica Ernesto Guevara porque ambos carecen de funcionalidades específicas para procedimientos y protocolos usados en estos laboratorios tales como los protocolos usados en los módulos agua-vapor, combustibles-lubricantes, etc. Además, estos sistemas no permiten la inclusión de nuevos módulos de desarrollo. Adicionalmente, un aspecto relevante es que el empleo de estos sistemas constituiría gastos para el país, que tiene como prioridad reducir costos en todas las áreas de producción. Todos estos elementos llevaron a la necesidad de desarrollar una solución propia,

("Traje a la Medida") de acuerdo con los requerimientos del Cliente.

Actualmente en el laboratorio de química de la Central Termoeléctrica Ernesto Guevara el registro de la actividad analítica se realiza utilizando documentos de textos, y hojas de cálculos. Esto trae consecuencia la generación de grandes volúmenes de información, difíciles de guardar y procesar lo que dificulta una buena explotación de los datos. Para dar solución al problema, se plantea como objetivo general: Desarrollar un sistema informático para la gestión de los ensayos fisicoquímicos de la Central Termoeléctrica Ernesto Guevara.

\section{Materiales y métodos.}

Actualmente el registro de la actividad analítica en el Departamento de Química comienza cuando el analista principal extrae reactivos del almacén de acuerdo a las necesidades de los ensayos diarios. Luego los analistas preparan un conjunto de soluciones valorantes y patrones con el objetivo de realizar ensayos fisicoquímicos destinados a evaluar las características de las aguas en los procesos de tratamiento de agua, aguas de calderas y las propiedades fisicoquímicas de los combustibles y lubricantes. Una vez obtenidos los resultados de los ensayos, estos se registran en modelos físicos que son analizados por los tecnólogos y el personal de explotación de la CTE. El análisis de dichos resultados permite evaluar la calidad y la seguridad del trabajo que se lleva a cabo y a su vez es la guía para tomar decisiones inmediatas sobre los procesos que tienen lugar en las diferentes áreas. Estos resultados a su vez son archivados para posteriores estudios que permitirán aumentar el conocimiento y la eficacia del proceso de producción de electricidad. Otro procedimiento que tiene lugar en el Departamento de Química es el control sobre el almacén de reactivos, el cual es realizado por auditores que seleccionan un diez por ciento de la documentación total para su revisión.

A partir del estudio y el análisis de los principales procesos a automatizar que se llevan a cabo en el Departamento de Química de la CTE Ernesto Guevara se ha elaborado el siguiente Diagrama de Casos de Uso (ver figura 1). 
Figura 1. Diagrama de Casos de Uso del Negocio.
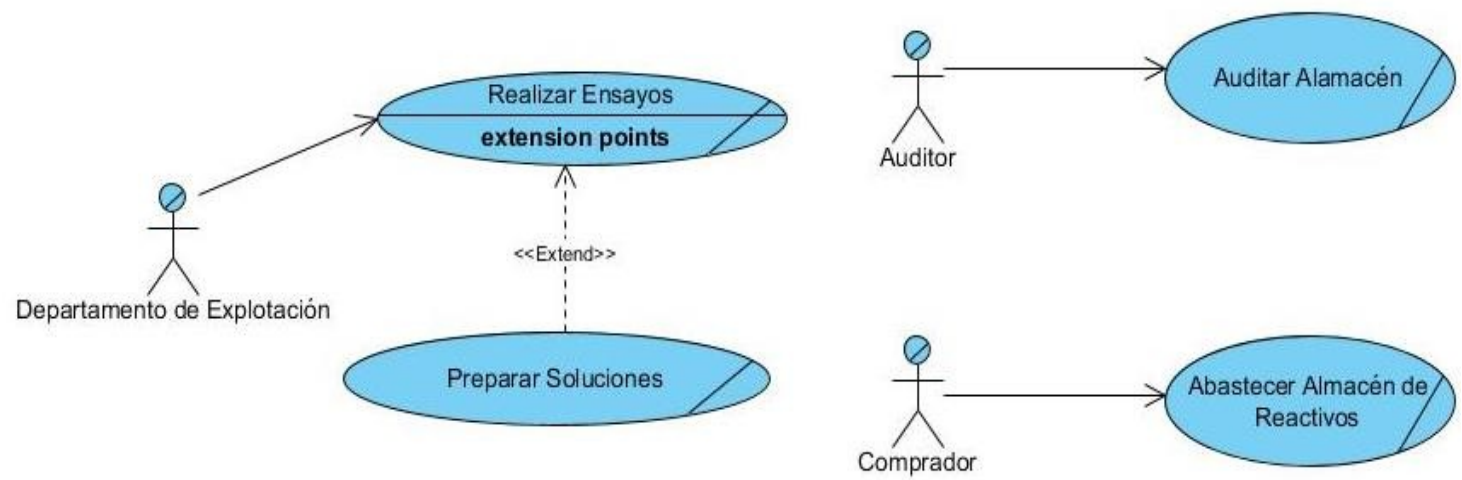

Fuente: Elaboración propia.

En la siguiente (tabla 1, 2, 3 y 4) se muestra la descripción textual de los casos de uso del negocio: Realización de Ensayos, Preparar Soluciones, Auditar Almacén y Abastecer Almacén de Reactivos.

Tabla 1. Descripción textual del caso de uso del negocio: Realizar Ensayos.

Nombre del caso de uso

Realizar Ensayos

\section{Actor}

Trabajadores

Resumen

\section{Acción del actor}

1. El Departamento de Explotación solicita ensayos.

\section{Departamento de Explotación}

Analista, Jefe de Equipo

El caso de uso comienza cuando el Departamento de Explotación solicita un conjunto de ensayos para evaluar la calidad, la seguridad y la eficiencia del proceso de producción eléctrica. El analista solicita al jefe de equipo los protocolos correspondientes, luego revisa la existencia de las soluciones necesarias para la realización de los ensayos y procede a realizarlos. El caso de uso termina cuando el analista envía los resultados obtenidos al Departamento de Explotación.

\section{Descripción del flujo normal de eventos}

\section{Respuesta del negocio}

2. El analista recibe la solicitud de ensayos.

3. El analista solicita los protocolos para los ensayos al jefe de equipo.

4. El jefe de equipo le facilita al analista los protocolos necesarios.

5. El analista chequea los protocolos. 
6. El analista verifica la existencia de las soluciones necesarias para realizar los ensayos.

7. El analista ejecuta los ensayos.

8. El analista registra los resultados de ensayos en el registro correspondiente.

9. El analista envía los resultados obtenidos al Departamento de Explotación.

10. El Departamento de Explotación recibe los resultados y los analiza.

11. El Departamento de Explotación registra los resultados.

Cursos alternos

Escenario: Faltan las soluciones necesarias.

6. Si el analista no encuentra las soluciones necesarias para la ejecución de los ensayos solicita al analista principal los reactivos para preparar las soluciones.

(Ver CU extendido Preparar Soluciones)

Fuente: Elaboración propia.

Tabla 2. Descripción textual del caso de uso del negocio: Preparar Soluciones.

Nombre del caso de uso

Preparar Soluciones

\section{Actor}

Trabajadores

Analista, Analista Principal

Resumen

El caso de uso inicia cuando el analista solicita al analista principal los reactivos necesarios, luego consulta las instrucciones para la preparación de las soluciones, y las ejecuta. El caso de uso termina cuando el analista imprime las etiquetas para las soluciones y las registra en el libro de soluciones.

\section{Descripción del flujo normal de eventos}

\section{Acción del actor}

\section{Respuesta del negocio}

1. El analista solicita al analista principal un conjunto de reactivos.

2. El analista principal busca los reactivos solicitados.

3. El analista principal entrega los reactivos solicitados.

4.El analista revisa las instrucciones para preparar la solución.

5.El analista prepara las soluciones.

6. El analista registra las soluciones preparadas e imprime las etiquetas necesarias para su identificación. 
ISSN: 2773-7330

Cursos alternos

Escenario: No existe los reactivos necesarios.

2. El analista principal busca los reactivos solicitados y no los encuentra.

3. El analista principal rechaza la solicitud de reactivos y realiza un pedido de estos al Departamento de Compras.

Fuente: Elaboración propia.

Tabla 3. Descripción textual del caso de uso del negocio: Auditar Almacén.

\begin{tabular}{|c|c|c|}
\hline Nombre del caso & \multicolumn{2}{|c|}{ Auditar Almacén } \\
\hline Actor & \multicolumn{2}{|l|}{ Auditor } \\
\hline Trabajadores & \multicolumn{2}{|c|}{ Analista Principal } \\
\hline Resumen & \multicolumn{2}{|c|}{$\begin{array}{l}\text { El caso de uso comienza cuando el auditor le solicita al analista principal el } \\
\text { inventario del almacén. Luego que el auditor recibe el inventario realiza un } \\
\text { muestreo sobre las operaciones realizadas en esta área. Más tarde realiza las } \\
\text { observaciones pertinentes a la audición y las registra en el libro de muestreos. }\end{array}$} \\
\hline \multicolumn{3}{|c|}{ Descripción del flujo normal de eventos } \\
\hline \multicolumn{2}{|l|}{ Acción del actor } & Respuesta del negocio \\
\hline \multicolumn{2}{|c|}{$\begin{array}{l}\text { 1. El auditor solicita el inventario de almacén } \\
\text { de sustancias. }\end{array}$} & 2. El analista principal entrega inventario a auditor. \\
\hline \multicolumn{2}{|c|}{ 3. El auditor revisa el inventario. } & \\
\hline \multicolumn{2}{|c|}{$\begin{array}{l}\text { 4. El auditor realiza las observaciones } \\
\text { pertinentes y las registra en el libro de } \\
\text { muestreos. }\end{array}$} & \\
\hline \multicolumn{3}{|c|}{\begin{tabular}{l|l} 
Cursos alternos & Escenario: \\
\end{tabular}} \\
\hline
\end{tabular}

Fuente: Elaboración propia

Tabla 4. Descripción textual del caso de uso del negocio: Abastecer Almacén de Reactivos

Nombre del caso de uso

\section{Actor}

Trabajadores

Resumen
Abastecer Almacén de Reactivos

\section{Comprador}

Analista Principal

El caso de uso comienza cuando el comprador realiza una entrega de reactivos al almacén, registra dicha operación en el registro de entregas, luego el analista principal recibe los reactivos y registra la acción en el archivo de entradas. Después, busca los reactivos abastecidos, y realiza los aumentos en las tarjetas pertinentes, si no encontrara alguno de los reactivos abastecidos crea una nueva tarjeta, y así termina el caso de uso.

\section{Descripción del flujo normal de eventos}


Acción del actor

1. El comprador realiza una entrega de reactivos al analista principal y hace contar la actividad en el registro de entrada.

Cursos alternos

\section{Respuesta del negocio}

2. El analista principal recibe los reactivos abastecidos.

3. El analista principal registra la entrega en el registro de entrada de reactivos.

4. El analista principal realiza una búsqueda en el inventario.

5. El analista principal aumenta la cantidad de reactivos en inventario.

Escenario: No encuentra algunos de los reactivos en el abastecimiento.

4.El analista realiza una búsqueda en el almacén de reactivos y no encuentra alguno de los reactivos abastecidos.

5.El analista principal crea una tarjeta de operaciones para el nuevo reactivo.

Fuente: Elaboración propia.

\section{Definición de los requisitos funcionales.}

Los requisitos funcionales son declaraciones de servicios que el sistema debe proporcionar, cómo debe reaccionar a entradas particulares y cómo debe comportarse en situaciones particulares (Sommerville; 2011).

A continuación, se exponen los requisitos funcionales según Sánchez (2019):

\section{RF1: Autentificación}

\section{RF2: Gestionar Usuarios}

RF\#2.1: Registrar usuario

RF\#2.2: Modificar usuario

RF\#2.3: Buscar usuario

RF\#2.4: Eliminar usuario

RF\#2.5: Listar usuario

\section{RF3: Gestionar Ensayos}

RF\#3.1: Registrar ensayos 
RF\#3.2: Modificar ensayos

RF\#3.3: Buscar ensayos

RF\#3.4: Eliminar ensayos

\section{RF4: Gestionar Protocolos de Ensayos}

RF\#4.1: Adicionar protocolo de ensayo

RF\#4.2: Modificar protocolo de ensayo

RF\#4.3: Buscar protocolo de ensayo

RF\#4.4: Eliminar protocolo de ensayo

RF\#4.5: Listar protocolo de ensayo

\section{RF5: Gestionar Soluciones}

RF\#5.1: Registrar soluciones

RF\#5.2: Modificar soluciones

RF\#5.3: Buscar soluciones

RF\#5.4: Eliminar soluciones

RF\#5.5: Listar soluciones

\section{RF6: Gestionar Equipos}

RF\#6.1: Registrar equipo

RF\#6.2: Modificar equipo

RF\#6.3: Buscar equipo

RF\#6.4: Eliminar equipo

RF\#6.5: Listar equipo

\section{RF7: Emitir Reportes del Almacén}

RF\#7.1: Emitir reportes de reactivos existentes en almacén

RF\#7.2: Emitir reportes de reactivos con cantidad mínima

RF8: Emitir Reportes 
RF\#8.1: Emitir reportes de ensayos realizados por analistas

RF\#8.2: Emitir reportes de ensayos realizados por tipo de ensayos

RF\#8.3: Graficar resultados de ensayos realizados por tipo de ensayo y por año

\section{RF9: Gestionar Reactivos en el Almacén}

RF\#9.1: Registrar reactivos

RF\#9.2: Modificar reactivos

RF\#9.3: Buscar reactivos

RF\#9.4: Eliminar reactivos

RF\#9.5: Listar reactivos

RF\#9.6: Aumentar cantidad de reactivo

RF\#9.7: Disminuir cantidad de reactivo

RF10: Gestionar Categorías de Reactivos

RF\#10.1: Registrar categoría

RF\#10.2: Modificar categoría

RF\#10.3: Buscar categoría

RF\#10.4: Eliminar categoría

RF\#10.5: Listar categoría

RF11: Gestionar Métodos

RF\#11.1: Adicionar métodos

RF\#11.2: Modificar métodos

RF\#11.3: Buscar métodos

RF\#11.4: Eliminar métodos

RF\#11.5: Listar métodos

RF12: Gestionar Normas de Ensayos

RF\#12.1: Adicionar normas 
RF\#12.2: Modificar normas

RF\#12.3: Buscar normas

RF\#12.4: Eliminar normas

RF\#12.5: Listar normas

\section{RF13: Gestionar Auditoría}

RF\#13.1: Registrar auditoría

RF\#13.2: Modificar auditoría

RF\#13.3: Buscar auditoría

RF\#13.4: Listar auditoría

En la figura 2 se muestra el modelo de casos de uso del sistema permitiendo que los desarrolladores de software y los clientes lleguen a un acuerdo sobre los requisitos, es decir, sobre las condiciones y posibilidades que debe cumplir el sistema. Este proporciona la entrada fundamental para el análisis, el diseño y las pruebas según (Booch y Rumbaugh; 2005).

Figura 2. Diagrama de casos de uso del sistema

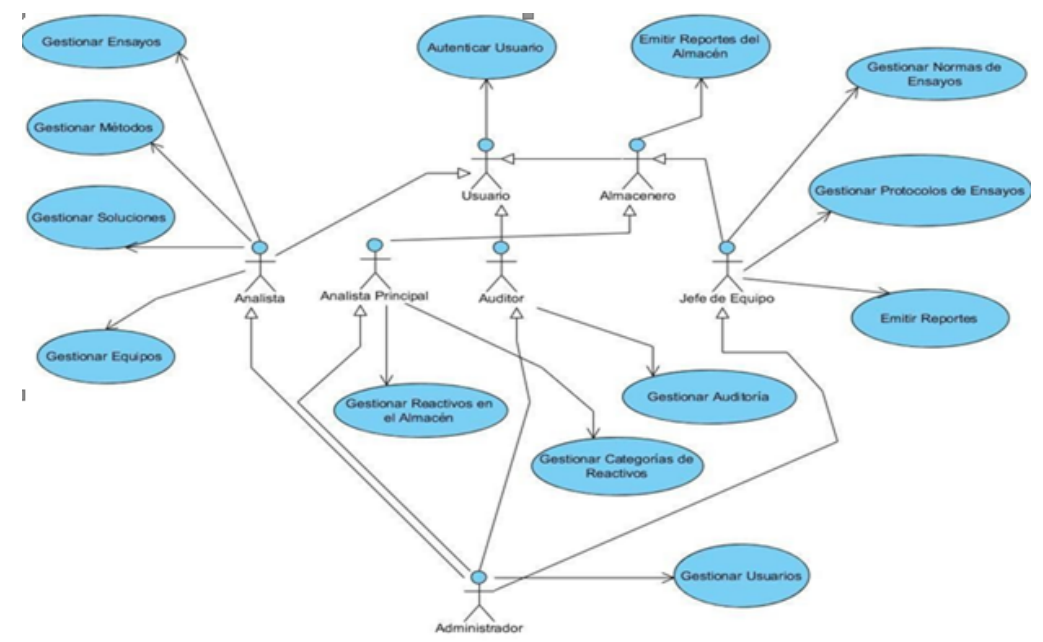

Fuente: Elaboración propia.

Los requisitos no funcionales, como su nombre indica, son requisitos que no están directamente relacionados con los servicios específicos entregados por el sistema a sus usuarios. Pueden relacionarse con propiedades de sistemas emergentes como la confiabilidad, el tiempo de respuesta y la ocupación de almacenamiento. Alternativamente, pueden definir restricciones en la implementación del sistema, tales como las capacidades de 
los dispositivos o las representaciones de datos utilizadas en interfaces con otros sistemas (Sommerville; 2011).

\section{Apariencia e Interfaz.}

La interfaz del sistema es simple e intuitiva para el uso, con una iconografía grande según especificaciones del cliente. Se usan colores claros y mantiene el mismo formato a través de todas las interfaces.

\section{Usabilidad.}

El sistema podrá ser usado por cualquier persona que posea conocimientos básicos en el manejo de la computadora.

\section{Extensibilidad.}

El sistema permite agregar nuevas funcionalidades y cambios en correspondencia con las exigencias de la entidad.

\section{Confiabilidad.}

La información está protegida contra accesos no autorizados, utilizando mecanismos de autenticación, la asignación de roles, y cifrado de claves a través del algoritmo bcrypt de Symfony. Todo lo mencionado anteriormente garantiza el cumplimiento estricto de medidas de seguridad.

\section{Software.}

Para el uso del sistema se necesitarán navegadores tales como Mozilla Firefox 30.0 o superior, Google Chrome v.35 o superior, Opera 36, o Microsoft Edge. Del lado del servidor, el sistema gestor de base de datos MySQL 5.7.23, el servidor web Apache 2.4.35 o superior, PHP 7 como mínimo y los sistemas operativos con soporte multiplataforma.

\section{Hardware.}

Del lado del cliente se puede emplear cualquier hardware con más de 1 GB de RAM y un espacio mínimo de 5 GB para almacenar la información que se solicita. Para el servidor, se debe emplear como mínimo un procesador Intel Dual Core a 3.0 GHz, 2 GB de RAM y 50 GB como mínimo de espacio libre en disco para guardar la información. También, para el funcionamiento del sistema se necesita una red estable con un ancho de banda mínimo 100 Mbits/s.

\section{Resultados y discusión.}

Descripción detallada del funcionamiento del sistema propuesto. 
El sistema está diseñado para gestionar la información relacionada con los ensayos fisicoquímicos que se llevan a cabo en la CTE Ernesto Guevara. Los usuarios, según sus roles y permisos podrán acceder y/o manipular la información de acuerdo con sus niveles de acceso al sistema. Para acceder al sistema el usuario tiene que autenticarse, si está autorizado se inicia la sesión y se muestra la página principal. El usuario con rol analista gestionará la información asociada a los ensayos, las soluciones, los métodos, los equipos. El usuario con rol analista principal gestionará la información relacionada con los reactivos en almacén, y las categorías para caracterizar las sustancias. El usuario con rol auditor tendrá permiso de visualizar el almacén de reactivos y gestionar la información de auditorías (inserta auditoría). El usuario con rol jefe de equipo tendrá la facultad de gestionar las normas para ensayos, los protocolos de ensayos y emitir reportes. Por último, el usuario con rol administrador podrá realizar todas las operaciones antes descritas y además gestionará la información asociada a los usuarios y roles del sistema.

En la figura 3 se muestra la interfaz de autenticarse donde el usuario del sistema pone su nombre usuario y contraseña para acceder a los diferentes componentes del software, si las credenciales no son correctas el usuario no logrará entrar al sistema en el caso contrario el usuario queda autenticado.

Figura 3. Autenticar usuario.

\section{Autenticarse}

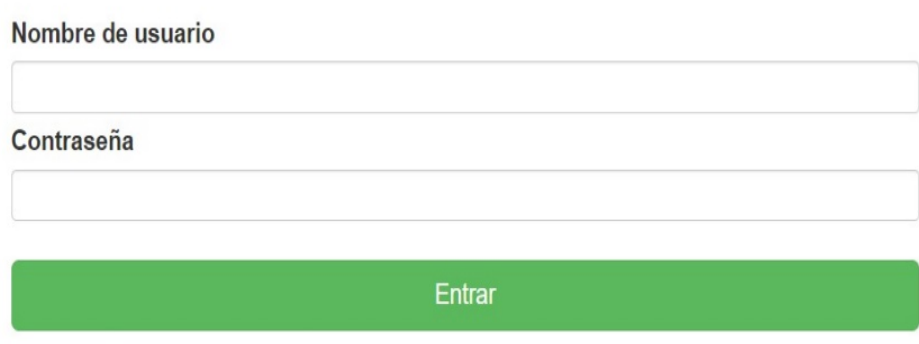

Fuente: Elaboración propia.

Una vez autenticado en el sistema si el usario es administrador puede gestionar la información de todos los uusarios del sistema como muestra la figura 4. Aquí el administrador de acuerdo con sus requerimientos registra, elimina, o modifica la información asociada a los usuarios. 
Figura 4. Gestionar usuarios por el administrador

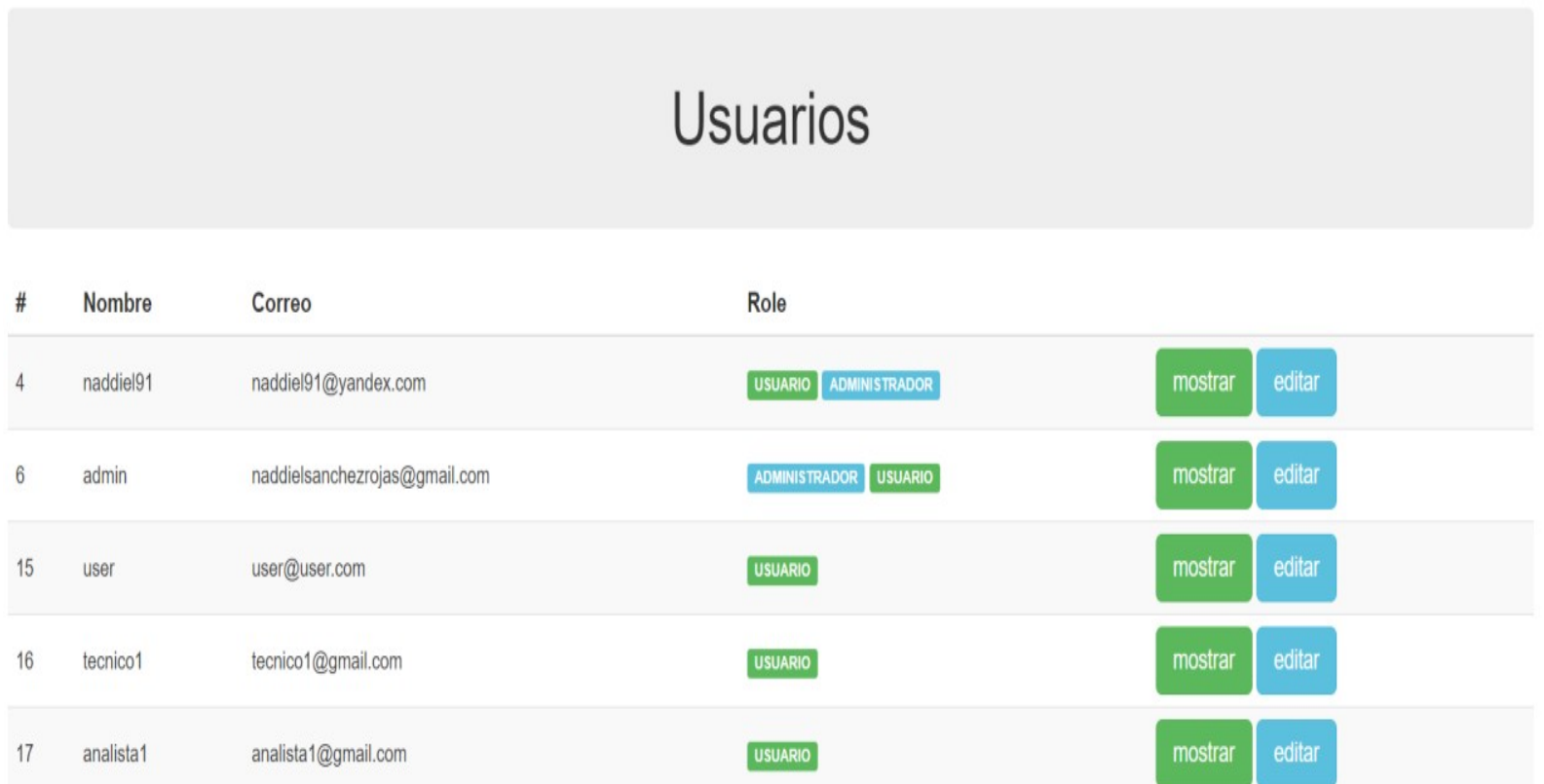

Fuente: Elaboración propia.

La figura 5 visualiza la interfaz de registrar las categorías de los reactivos donde el analista principal necesita registrar, editar, filtrar y eliminar las categorías de los reactivos del almacén. El analista debe estar previamente autenticado con los permisos correctos.

Figura 5. Gestionar las categorías de reactivos.

\section{Categorías para Reactivos}

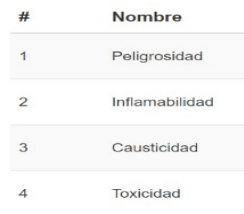

\begin{tabular}{|c|c|c|}
\hline mostrar & editar & Eliminar \\
\hline mostrar & editar & Eliminar \\
\hline mostrar & editar & Eliminar \\
\hline mostrar & editar & Ellminar \\
\hline
\end{tabular}

Fuente: Elaboración propia.

Después de gestionada las categorías, el analista necesita gestionar los métodos de ensayos como se muestra en el figura 6. En ella se muestra la interfaz Métodos que contiene un listado de diferentes métodos donde puede hacer las diferentes acciones como: registrar, modificar, buscar y eliminar. 
Figura 6. Gestionar los métodos asociados a los ensayos.

\section{Métodos}

\begin{tabular}{lllll}
$\#$ & Nombre & & \\
\hline 1 & Colorimétrico & mostrar & editar & Eliminar \\
\hline 3 & Volumétrico & mostrar & ediltar & Eliminar \\
\hline 3 & Potenciométrico & mostrar & edilar & Eliminar \\
\hline 4 & Gravimétrico & mostrar & ediltar & Eliminar \\
\hline
\end{tabular}

Fuente: Elaboración propia.

Cuando el usuario es el jefe del equipo y necesita gestionar las normas del ensayo, el sistema muestra la interfaz del listado de normas (ver figura 7). De acuerdo con las operaciones a realizar el jefe de equipo puede adicionar, modificar, buscar y eliminar.

Figura 7. Gestionar las normas asociadas a los ensayos.

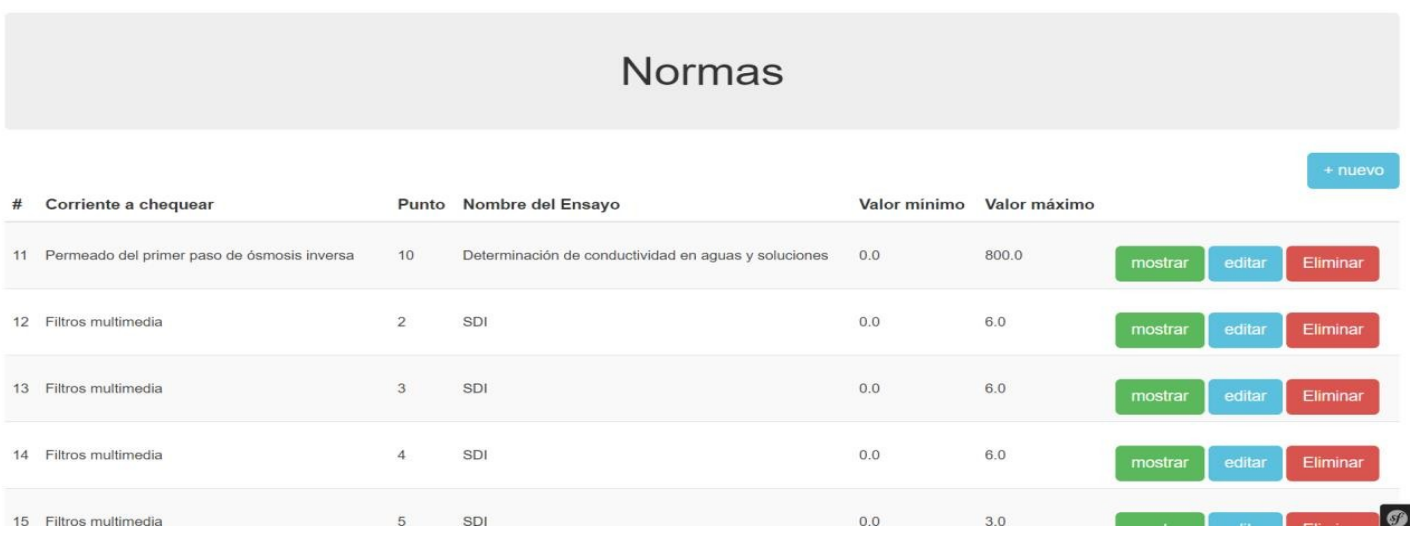

Fuente: Elaboración propia

Otro componente muy importante en el software son los reportes (ver figura 8), donde el jefe de equipo debe estar previamente autenticado con los permisos correctos y debe visualizar la interfaz Repores que muestra los tipos de reportes a consultar y/o exportar y seleccionar las operaciones a realizar. 
Figura 8. Reportes relacionados con la actividad analítica del Departamento de Química.

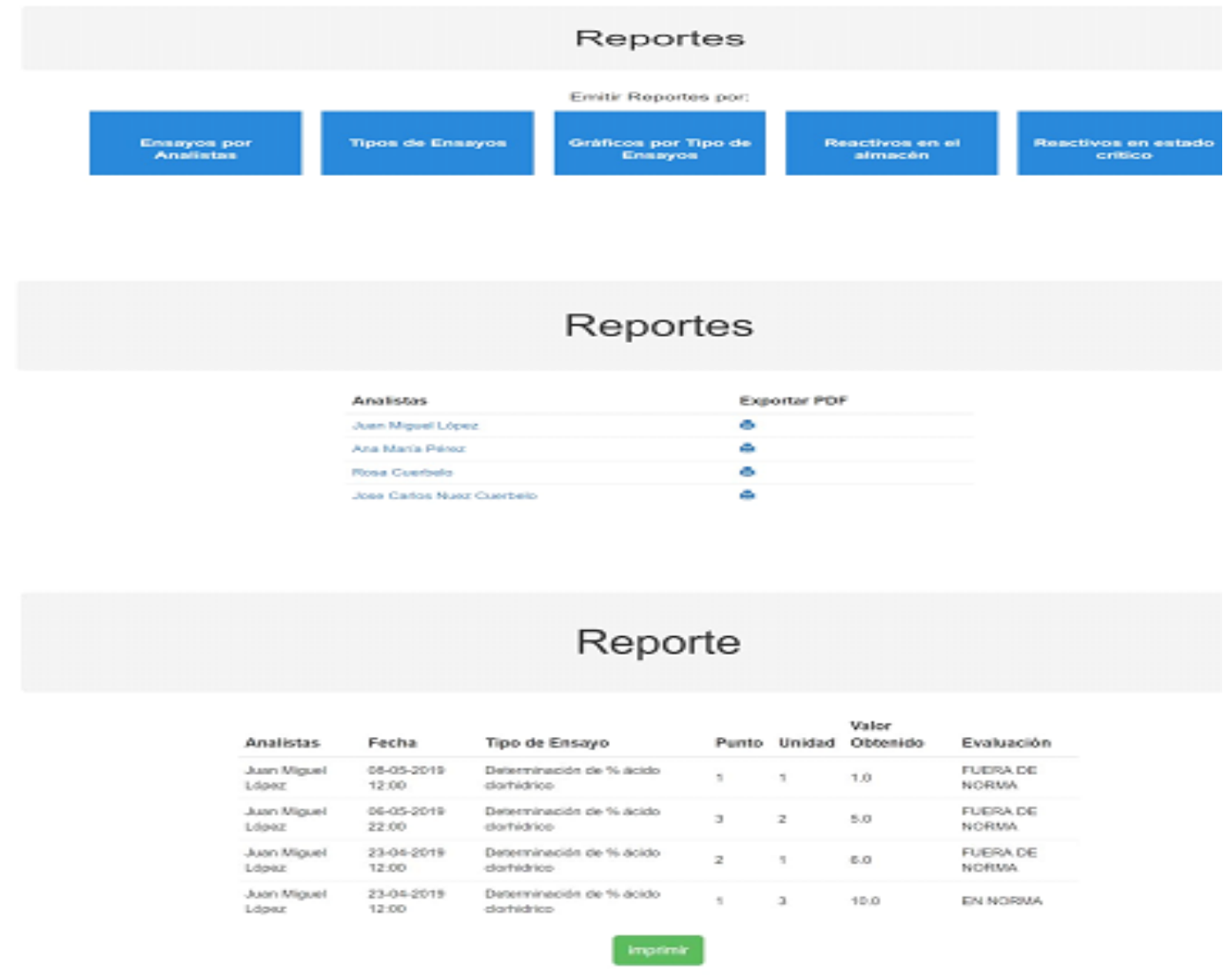

Fuente: Elaboración propia.

\section{Conclusiones.}

- El estudio de la información generada durante los ensayos realizados posibilitó identificar que los principales procesos a automatizar eran la gestión de los ensayos, la gestión de soluciones, así como la gestión de los reactivos necesarios para ello.

- A partir de la identificación de los procesos a automatizar y el levantamiento de requisitos realizado se llevó a cabo el diseño del sistema informático utilizando una arquitectura Modelo-Vista-Controlador.

- Tomando como base el diseño obtenido y las herramientas y tecnologías a utilizar, se realizó la implementación de un sistema informático que permite organizar y consultar con rapidez la información relacionada con los ensayos físicoquímicos. 


\section{Referencias bibliográficas.}

Booch, G y Rumbaugh, J (2005). The Unified Modeling Language User Guide. Covers UML.Editorial 978-0. ISBN:978-0-321-26797-9.p.476

ECURED (2018). Historia de la Electrificación. Disponible en: www.ecured.cu/Historia_de_la_electrificación.

Jiong, S (2015).Steam power plant configuration, design, and control. WIREs Energy Env.

Mansoor-ul, H (2019). International Technical Sciences Journal. Power Gener Methods Tech Econ Strategy, junio.

Sommerville, I (2011). Software Engineering. Editorial Addison-Wesley.ISBN:978-0-20139815-1.693p.

Sánchez, N.J (2019). Sistema informático para la gestión de ensayos fisicoquímicos en la Central Termoeléctrica Ernesto Guevara. Tesis en opción al título de Ingeniero en Informática. Facultad de Ciencias Técnicas. Universidad Agraria de la Habana. Mayabeque. 148p.

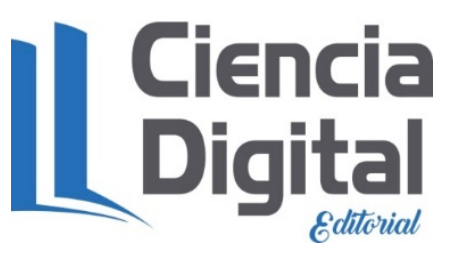




\section{Para citar el artículo indexado}

Sánchez Rojas, N. J., Machín Armenteros, A., \& Marrero García, Y. (2020). Sistema informático para la gestión de ensayos fisicoquímicos en la Central Termoeléctrica Ernesto Guevara. AlfaPublicaciones, 2(2), 29-46. https://doi.org/10.33262/ap.v2i2.27

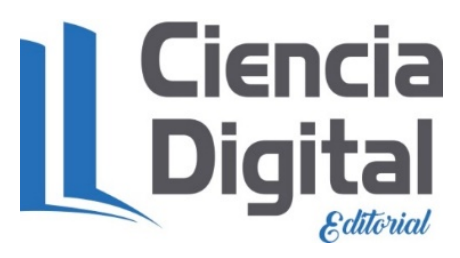

El artículo que se publica es de exclusiva responsabilidad de los autores y no necesariamente reflejan el pensamiento de la Revista Alpha Publicaciones.

El artículo queda en propiedad de la revista y, por tanto, su publicación parcial y/o total en otro medio tiene que ser autorizado por el director de la Revista Alpha Publicaciones.
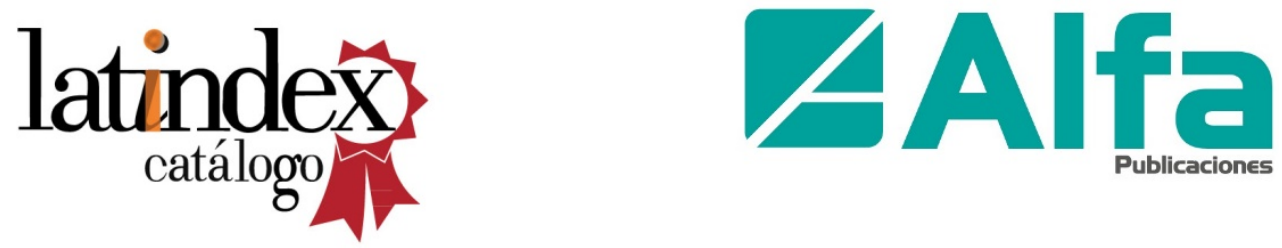